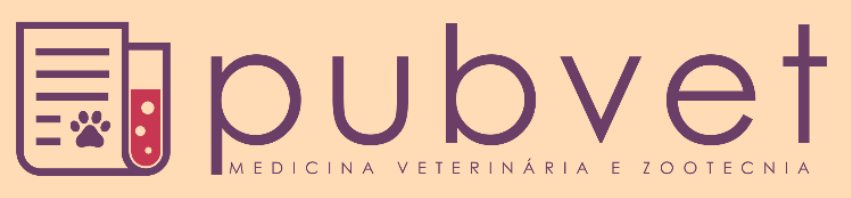

https://doi.org/10.31533/pubvet.v13n12a471.1-10

\title{
Alimentos alternativos na nutrição de coelhos: Revisão
}

\author{
Alison Batista Vieira Silva Gouveia ${ }^{* \bullet}$, Lorrayne Moraes de Paulo ${ }^{2} \bullet$, Júlia Marixara Sousa da Silva ${ }^{1} \bullet$, \\ Weslane Justina da Silva ${ }^{3}$, Janaína Borges dos $\operatorname{Santos}^{4}{ }^{\circ}$,Stéfane Alves Sampaio ${ }^{4}{ }^{\circ}$, Elísio Marques de \\ Almeida Júnior ${ }^{4} \theta$, Karine Oliveira $\operatorname{Costa}^{4} \theta$, Jiovanna Gonçalves de Sousa ${ }^{4} \theta$, Fabrício Eumar de \\ Sousa $^{50}$, Adriano Carvalho $\operatorname{Costa}^{60}$, Fabiana Ramos dos $\operatorname{Santos}^{60}$, Cibele Silva Minafra ${ }^{60}$ \\ ${ }^{I}$ Doutorando do Programa de Pós-Graduação em Zootecnia, Universidade Federal de Goiás, Escola de Veterinária e Zootecnia, Campus \\ Samambaia, Goiânia, GO, Brasil. \\ ${ }^{2}$ Mestranda do Programa de pós-graduação em zootecnia, Universidade Federal de Goiás, Escola de Veterinária e Zootecnia, Campus \\ Samambaia, Goiânia, GO, Brasil. \\ ${ }^{3}$ Mestra pelo Instituto Federal de Ciência e Tecnologia Goiano, Departamento de Zootecnia. Campus Rio Verde, GO, Brasil \\ ${ }^{4}$ Graduanda em zootecnia pelo Instituto Federal de Ciência e Tecnologia Goiano, Departamento de Zootecnia. Campus Rio Verde, GO, Brasil. \\ ${ }_{5}^{5}$ Professor do Centro Universitário de Mineiros, UNIFIMES, Departamento de Agronomia, Mineiros, GO, Brasil. \\ ${ }^{6}$ Professores do Instituto Federal de Ciência e Tecnologia Goiano, Departamento de Zootecnia. Campus Rio verde, GO, Brasil \\ *Autor para correspondência, E-mail: alisonmestre28@gmail.com
}

Resumo. Na alimentação animal as dietas são formuladas visando proporcionar totais condições para os expressarem todo seu potencial produtivo contudo maior parte dos ingredientes utilizados para compor as dietas balanceadas aumentam o custo do produto final. O objetivo desta revisão é evidenciar quais os alimentos alternativos são disponíveis em território brasileiro com potencial uso para a serem utilizados na nutrição de coelhos. Percebeu-se que os principais alimentos alternativos utilizados são glicerina bruta, casca de soja, pólen apícola, polpa cítrica desidratada, farelo de algodão e coprodutos da canade-açúcar. Neste sentido, a utilização destes produtos secundários proporciona maior sustentabilidade na produção cunícula sem proporcionar influências negativas sobre o desempenho ou sobre o metabolismo destes animais, auxiliando na redução os custos de produção com alimentação.

Palavras chave: Cecotrofia, cunicultura, fibra

\section{Alternative Foods in Rabbit Nutrition: Review}

Abstract. In animal feed diets are formulated to provide total conditions to express their full productive potential, however most of the ingredients used to make balanced diets consequently increase the cost of the final product. The objective of this review is to show which alternative foods are available in Brazilian territory with potential use to be used in rabbit nutrition. It has been found that the main alternative foods used are crude glycerin, soybean hulls, bee pollen, dehydrated citrus pulp, cottonseed meal and co-products from sugarcane. In this sense, the use of these by-products provides greater sustainability in the production without affecting negative performance or metabolism of these animals, helping to reduce the costs of production with feed.

Keywords: Cecotrophy, rabbit breeding, fiber

\section{Alimentos alternativos en la nutrición de conejos: Revisión}

Resumen. En la alimentación animal las dietas se formulan para proporcionar totales condiciones para expresar todo su potencial productivo, pero la mayor parte de los ingredientes utilizados para componer las dietas balanceadas aumentan el costo del producto final. El objetivo de esta revisión es evidenciar qué alimentos alternativos están disponibles 
en territorio brasileño con potencial uso para ser utilizados en la nutrición de conejos. Se percibió que los principales alimentos alternativos utilizados son glicerina bruta, cáscara de soja, polen apícola, pulpa cítrica deshidratada, salvado de algodón y coproductos de la caña de azúcar. En este sentido, la utilización de estos productos secundarios proporciona una mayor sostenibilidad en la producción de la cunícula sin proporcionar influencias negativas sobre el rendimiento o sobre el metabolismo de estos animales, ayudando a reducir los costos de producción con alimentación.

Palabras clave: Cecotrofia, cunicultura, fibra

\section{Introdução}

O Brasil é conhecido por seu grande potencial agrícola, devido às grandes extensões de terra favorecendo os vários tipos de culturas da atividade zootécnica, incluindo a cunicultura. Esta atividade, em geral, é pouco conhecida e sua difusão é baixa, considerando-se seu grande potencial e importância para o desenvolvimento urbano e rural (Valentim et al., 2018). No entanto, esta atividade, passou e ainda passa por inúmeras dificuldades relacionadas à tecnologia de produção, além da deficiência organizacional na cadeia produtiva e falta de políticas específicas para o setor o que eleva o custo de produção. A estruturação adequada da cadeia produtiva, bem como, a promoção do diálogo entre os criadores é fundamental para que a atividade seja exercida de maneira mais segura e rentável (Machado \& Ferreira, 2014).

A redução dos custos com a alimentação na cunicultura é um fator central para intensificar esta atividade. Neste sentido, é necessário avaliar ingredientes alternativos que possam substituir ingredientes convencionais, reduzindo o preço da ração e colaborando para que haja maior lucratividade nesta atividade (Molina et al., 2015). Segundo Klinger \& Toledo (2016) em comparação a outras espécies, e pequena a utilização de subprodutos em dietas para coelhos no Brasil, contudo novos ingredientes vêm mostrando grande capacidade nutricional para serem utilizados na alimentação cunícula, melhorando a lucratividade do produtor. Todavia, devem ser observados possíveis efeitos negativos do uso destes alimentos que podem comprometer o desempenho dos animais e também outras características zootécnicas.

Os coelhos são conhecidos por sua fecundidade e prolificidade, pela habilidade em utilizar forragens e pelos baixos níveis de gordura e colesterol em suas carnes. Entretanto, esses animais, especialmente os recém-desmamados, não devem ser mantidos somente com forragens. $\mathrm{O}$ uso de rações garante a máxima produtividade; porém, o alto custo dos ingredientes devido à competição entre o homem e os animais pelos grãos é um dos problemas para a produção animal (Oliveira \& Pádua, 2010). Machado \& Ferreira (2012) relatam que os coelhos são animais herbívoros que necessitam de uma dieta equilibrada de acordo com suas necessidades nutricionais. É de extrema importância que se tenha pleno conhecimento do valor nutricional das matérias primas disponíveis na região, bem como da utilização de aditivos e alimentos funcionais.

O objetivo desta revisão foi obter conhecimento sobre a nutrição de coelhos bem como alimentos alternativos que são utilizados na alimentação destes animais.

\section{Particularidades anatômicas dos coelhos}

Uma das características peculiares do coelho em relação a outros mamíferos monogástricos é o aproveitamento da celulose. Este fato se deve a microflora contida no intestino grosso deste lagomorfo que são capazes de quebrar alguns polímeros contidos na parede vegetal, possibilitando o seu aproveitamento (Paula et al., 2016). Segundo Coelho et al. (2016) outro aspecto que deve ser levado em consideração está relacionado aos processos de digestão microbiana no ceco, que permitem aos coelhos a utilização de quantidades significativas de alimentos volumosos nas rações, entre 40 a $50 \%$ da ração, para o atendimento das exigências nutricionais em fibra. De acordo com Arruda et al. (2003) o intestino grosso desempenha importante papel na digestão de coelhos, devido ao processo fermentativo cecal, a excreção seletiva de fibra pela região do ceco-cólon e a re-ingestão de conteúdo cecal ou cecotrofia, a qual faz parte da fisiologia e do equilíbrio digestivo de coelhos. Os coelhos possuem a característica de acomodar em seu trato digestivo uma população microbiana simbiótica, com funções digestivas nas 
quais o hospedeiro é incapaz de realizar, como a digestão de carboidratos estruturais, a síntese de aminoácidos essenciais e de vitaminas do complexo $\mathrm{B}$, permitindo a sobrevivência à base de rações de baixo valor nutricional como os alimentos fibrosos (Cheeke, 1995; De Blas \& Wiseman, 2010).

\section{Cecotrofia}

O coelho (Oryctolagus cuniculus) é um animal monogástrico de ceco funcional que realiza a cecotrofia que possibilita a ingestão de material altamente fibroso com sua concomitante fermentação no ceco, e transformação em cecotrófos. Os cecotrófos são para o coelho um alimento muito nutritivo, que satisfaz parte de sua exigência nutricional diária, portanto quaisquer alimentos ou dieta que possa vir a interferir nesse comportamento ou composto devem ser avaliados (Paula et al., 2016). A quantidade produzida de cecotrófos, assim como a sua contribuição nutricional, pode ser influenciada pelo nível e tipo de fibra que é o principal substrato para a atividade fermentativa cecal nos coelhos (Arruda et al., 2003). A composição nutricional dos cecotrófos é influenciada pela dieta, o que pode proporcionar uma maior quantidade de nutrientes. O consumo de cecotrófos é influenciado pela proteína e pela energia da dieta. Quando a dieta possui uma baixa quantidade de energia, a ingestão dos cecotrófos é maximizada (Jenkins, 1999).

\section{Fibra na alimentação de coelhos}

Os coelhos são herbívoros e monogástricos, cujos ingredientes da dieta consistem principalmente de fibras. Após o desmame o trato gastrointestinal não está totalmente desenvolvido, o que leva a redução da digestibilidade dos nutrientes e pode acarretar também doenças digestivas, portanto a qualidade da fibra alimentar e muito importante na nutrição de coelhos (Liao et al., 2017). Segundo Retore et al. (2010) muitos ingredientes alternativos apresentam em sua composição, além de proteína e energia, elevados teores de fibra dietética com diferentes graus de solubilidade e composição química, o que pode ser fator determinante na sua utilização pelo animal. Arruda et al. (2003) concluíram que a fibra participa especialmente como um "lastro" ao propiciar um adequado tempo de trânsito da digesta, e consequentemente, participa no fornecimento de energia para o animal via produtos da atividade microbiana cecal. A qualidade e a quantidade da fibra dietética influenciam a velocidade de passagem e no padrão fermentativo, sugerindo-se que alimentos fibrosos contendo baixa lignificação da parede celular aliado à maior proporção de celulose e hemicelulose ou pectinas, não devem ser empregados como única fonte de fibra em rações completas, pela susceptibilidade às diarreias fatais. A inclusão de níveis elevados de volumosos na dieta pode proporcionar maior peso relativo do sistema digestório, influenciado pelo maior nível de fibra e menor disponibilidade de nutrientes. A introdução desse programa de alimentação pode ser prejudicial ao tamanho do fígado dos animais bem como rendimento de carcaça (Machado \& Ferreira, 2016).

\section{Alimentos alternativos}

A procura por alimentos não convencionais aumentou nos últimos anos, principalmente devido aos frequentes aumentos nos preços de grãos de cereais e fontes proteicas vegetais (Coelho et al., 2016). O estudo dos alimentos alternativos procura dar subsídios para a produção de rações além de mais baratas, de mesma qualidade nutricional, proporcionando desempenho produtivo equivalente àquelas formuladas com alimentos convencionais (Galan et al., 2013). Segundo Ferreira et al. (2015) o interesse na adição de subprodutos da agroindústria em dietas para coelhos está focado na busca de ingredientes não competitivos com a alimentação humana, que promovam desempenho produtivo e rentabilidade satisfatórios, somando-se ao fato de possibilitar maiores opções de destino de resíduos potencialmente poluidores ao ambiente.

\section{Principais alimentos alternativos utilizados na alimentação de coelhos}

\section{Glicerina bruta}

De acordo com Gonçalves et al. (2014), o termo glicerina pode ser empregado para tratar de uma substância pura, podendo ser empregada a produtos comerciais que contenham em sua composição aproximadamente $95 \%$ de glicerol. Já o termo glicerol pode ser utilizado para definir um produto puro. 
A glicerina já foi estudada na alimentação de frangos de corte (Simon et al., 1996); porém, com o aumento da produção de biodiesel, e consequente a grande disponibilidade deste subproduto aumentou o interesse da utilização da glicerina na alimentação de coelhos, visando à redução dos custos de produção com a fonte energética da dieta (Beserra et al., 2016). A utilização da glicerina bruta na formulação de rações para monogástricos desperta interesse imediato, pois se constitui em um produto rico em energia dietética, fornecendo aproximadamente $3.696 \mathrm{kcal} / \mathrm{kg}$ de energia total, com metabolização de aproximadamente 95\% (Rostagno et al., 2017). De acordo com Dozier et al. (2008) esta alta taxa de metabolização se dá provavelmente em função de sua alta taxa de absorção devido ao baixo peso molecular e ao modo de absorção, por meio de difusão facilitada. A sua composição química está diretamente relacionada ao valor energético que a mesma irá apresentar (Bernardino et al., 2014).

Segundo Lehninger (2006) na ração a glicerina pode ser aproveitada, pois o glicerol absorvido no intestino e posteriormente transportado para o fígado, onde após serem realizadas todas as reações enzimáticas específicas, poderá ser utilizado metabolicamente para a síntese de glicose (gliconeogênese), síntese de lipídeos (lipogênese) ou ser completamente oxidada para a produção de energia via glicólise e ciclo de Krebs. Tendo que ser levado em consideração que a via na qual o glicerol será utilizado depende do estado energético do animal no momento considerado (Lehninger, 2006).

O uso da glicerina na alimentação animal vem sendo cogitado em virtude da disponibilidade desse coproduto e da possibilidade de seu emprego na alimentação animal como ingrediente que corrobora bons índices de desempenho e baixo custo (Cruz et al., 2014; Eiras et al., 2014; Eiras et al., 2013; Françozo et al., 2013). De acordo com Beserra et al. (2016) muitas pesquisas têm sido realizadas para verificar o real efeito da inclusão da glicerina na alimentação animal, e sua utilização têm apresentados resultados que demonstraram que a glicerina se apresenta como uma opção de ingrediente para a substituição do milho e outros grãos (fornecimento de energia) na dieta de coelhos, suínos, aves e ruminantes, tornando esse processo de produção mais atrativo e com menor custo de produção.

Luciano et al. (2017) concluíram que coelhos conseguem aproveitar melhor os nutrientes quando alimentados com dietas contendo glicerina. Contudo, é importante considerar que tanto o valor energético quanto o nível máximo de inclusão na ração podem variar em função da composição química e do grau de processamento da glicerina. Klinger et al. (2015) verificaram que a inclusão de glicerina bruta em dietas para coelhos na fase de crescimento, não altera o desempenho dos animais. Desta forma, a inclusão deste ingrediente em substituição aos ingredientes energéticos usualmente utilizados para coelhos nesta fase é viável até o nível de 7,5\%. Retore et al. (2012a) concluíram que coelhos na fase de crescimento alimentados com dietas contendo glicerina semi-purificada vegetal e mista apresentaram energia digestível de 4.048 e $3.697 \mathrm{kcal} / \mathrm{kg}$ MS, respectivamente, mostrando serem fontes altamente energéticas. A glicerina semi-purificada vegetal pode ser incluída em até $12 \%$ da dieta, e a semi-purificada mista, em até $9 \%$, sem afetarem o desempenho e peso de carcaça dos animais, além de reduzirem o custo de produção. Retore et al. (2012b) avaliaram a inclusão glicerina vegetal bruta e glicerina bruta mista em dietas para coelhos em crescimento, as quais apresentaram energia digestível de 5.099 e $4.953 \mathrm{kcal} / \mathrm{kg}$ de matéria seca. Glicerina bruta mista pode ser incluída no nível máximo de $12 \%$, enquanto glicerina vegetal bruta pode ser adicionada a $6 \%$ em dietas para coelhos em crescimento, analisando o custobenefício para avaliar o melhor nível a ser utilizado. Iñigo et al. (2011) concluíram que o glicerol bruto pode substituir o amido até níveis de 5\% em dietas para coelhos, sem efeito adverso ou benéfico sobre o consumo de ração, a eficiência alimentar e o desempenho produtivo e de lactação.

\section{Casca de soja}

A casca de soja é um subproduto da extração do óleo de soja, apresentando um elevado nível de fibra, um nível médio de proteína bruta e baixa energia, o que favorece a sua utilização para ruminantes e em rações que não necessitam de um alto teor de energia. Em monogástricos a sua utilização é restrita devido ao alto teor de fibra (Arruda et al., 2003). Esse co-produto se destaca pela elevada oferta, preços competitivos e composição bromatológicas que se adequa à alimentação cunícola; pois possui proporção de frações altamente fermentáveis (pectinas, hemiceluloses e celulose), associada à baixa presença de ligninas, um dos principais componentes que afeta negativamente a digestão de fibra (Zambom et al., 2001).

Segundo Nicodemus et al. (2007) ingredientes volumosos que apresentam maior concentração de polímeros não amilolíticos com menor teor de lignina, como a casca de soja, podem ser utilizados na dieta 
de coelhos pois serão degradados mais intensamente no intestino grosso, quando comparado a volumosos convencionais, devido à maior acessibilidade dos constituintes fermentáveis à microbiota cecocólica. Toledo et al. (2012) e Klinger et al. (2014) concluíram que a casca de soja pode ser incluída em dietas para coelhos em crescimento em até $100 \%$, pois mantém índices satisfatórios de desempenho e rendimento de abate, aliados à melhor viabilidade econômica. Os dados obtidos nestes estudos podem colaborar para o crescimento da cunicultura, mas ainda são necessários estudos mais aprofundados com a utilização deste coproduto em virtude da escassez de dados. Em pesquisas mais recente Shang et al. (2017) concluíram que a utilização da casca de soja pode ser realizada na se deita de coelhos de engorda até $200 \mathrm{~g} / \mathrm{kg}$, sem provocar efeitos adversos sobre o desempenho de crescimento, eficiência alimentar, características de carcaça e qualidade da carne.

\section{Polpa cítrica desidratada}

A polpa cítrica desidratada é um dos coprodutos das frutas cítricas, que é uma mistura de casca, polpa e sementes de frutas cítricas (Muller \& Prado, 2004). Uso de coprodutos agroindustriais como ração pode efetivamente diminuir o custo do processamento e gerenciamento de resíduos que tem sido um tema quente na indústria pecuária (Salvador et al., 2014).

As polpas obtidas do processamento de citros são, portanto, coprodutos relevantes em termos de quantidades produzidas e disponibilidade, de fácil inclusão em formulações de ração e de transporte por se tratar de um produto desidratado. Assim, o uso de polpa cítrica desidratada é cada vez mais interessante como fonte de energia para ruminantes, mas também para algumas espécies monogástricas, como coelhos (De Blas et al., 2018).

A polpa cítrica desidratada e uma fonte de fibra digestível, possuindo elevados níveis de pectina e reduzida lignificação, sendo bem aproveitada na forma de energia pelos coelhos, além de serem compostas de pequenas quantidades de fibra indigestível, características que aliam valor nutritivo a efeito lastro, podendo conter 20 a 40\% de açúcar e 22 a $44 \%$ de carboidratos de fibra solúvel em detergente (Maria et al., 2013; Muller \& Prado, 2004), podendo ter uma variação conforme e mostrado na Tabela 1.

Tabela 1. Composição de polpa cítrica seca (DCP)

\begin{tabular}{lcc}
\hline Polpa cítrica seca & Variação & Média \\
\hline Proteína (\%) & $5,0-9,3$ & 6,16 \\
Gordura (\%) & $1,3-9,1$ & 3,74 \\
Fibra Bruta (\%) & $6,4-16,8$ & 12,28 \\
Cinza (\%) & $3,1-8,4$ & 4,68 \\
Umidade (\%) & $3,5-13,7$ & 8,58 \\
\hline
\end{tabular}

Fonte: Alnaimy et al. (2017).

Segundo De Blas et al. (2018) a inclusão de polpa cítrica desidratada em rações para coelhos é limitada pelo seu baixo suprimento de proteína bruta e pelo alto nível de Ca. Os principais fatores de variação são o tipo de fruta (sendo o limão diferente dos demais) e o processamento (seco ou fresco). O método de desidratação, particularmente a adição de calcário e a temperatura de desidratação, podem afetar a composição da polpa cítrica seca. Contudo, estudos realizados utilizando a polpa cítrica desidratada na nutrição de coelhos em diferentes fases têm sido realizados com resultados satisfatórios. Até $25 \%$ de milho dietético em dieta de coelho pode ser substituído por polpa cítrica desidratada, sem efeitos negativos no desempenho de coelhos, como uma fonte de alta energia, sua inclusão na formulação de ração para coelhos ajudaria a reduzir o custo da ração. Ibrahim et al. (2011) verificaram que os efeitos benéficos das polpas cítricas desidratadas provenientes da laranja ou do limão podem promover a melhoria da digestibilidade e valor nutritivo dos nutrientes, além do elevado conteúdo de antioxidantes e ácidos graxos e essenciais (ômega3), podendo ser incorporados nas formulações de dietas saudáveis e econômicas. Lu et al. (2018) concluíram que a polpa cítrica pode ser usada como recurso alimentar disponível para coelhos, e a inclusão de até $20 \%$ de polpa cítrica desidratada na dieta de coelhos não apresenta efeitos adversos no desempenho do crescimento e ajudou a melhorar a concentração sérica de Ca e o estado antioxidante do fígado. 


\section{Farelo de algodão}

O Brasil possui uma grande produção de algodão e, consequentemente, grande disponibilidade dos subprodutos da indústria têxtil, a baixo custo. Dessa forma, o uso destes subprodutos na nutrição de coelhos, deve ser considerado, por possuírem grande potencial na diminuição dos custos de produção (Paim et al., 2010).

Dentre os farelos proteicos, o de algodão é o terceiro mais produzido no mundo, perdendo apenas para os de soja e os de canola. A composição química do farelo de algodão é altamente variável, dependendo da variedade plantada e da quantidade de cascas extraídas durante o processo. Assim, os farelos de algodão comercializados podem apresentar variações significativas no teor de proteína (30 a $40 \%$ ) e fibra bruta (11 a 22\%) (Dávila et al., 2007). Podendo apresentar fatores anti-nutricionais, como o gossipol (Santos et al., 2013), fator anti-nutricional prejudicial ao crescimento e reprodução dos animais, além de apresentar baixos teores de lisina e aminoácidos sulfurados. Sua inclusão deve ser cuidadosa e níveis de até 5\% podem ser utilizados (Rostagno et al., 2017). Dávila et al. (2007) concluíram que rações balanceadas para coelhos em crescimento, com farelo de algodão contendo $38 \%$ de PB pode substituir eficientemente o farelo de soja, ficando seu uso dependente das condições de mercado.

\section{Coprodutos da cana-de-açúcar}

A vinhaça é o principal coproduto da fabricação do etanol e da cachaça, apresenta coloração escura, odor adocicado, $\mathrm{pH}$ ácido, altos índices de demanda biológica de oxigênio, altos teores de compostos minerais, sobretudo de K, Ca, Mg, S e N (Hidalgo et al., 2009; Martins et al., 2017). A vinhaça tem sido utilizada na forma líquida na alimentação animal como aditivo por suas propriedades probióticas, como veículo em pré-misturas, palatabilizante ou promotora de maturação e reprodução sexual. Seu uso como aditivo geralmente resultou em melhor conversão alimentar e aumento do peso corporal e crescimento devido à presença dos ácidos orgânicos, que melhorar o uso de nutrientes, digestão, síntese de vitamina D e vitamina C e absorção mineral, que facilitam a alimentação metabolismo (Hidalgo et al., 2009; Martins et al., 2017). Oliveira et al. (2013) concluíram que vinhaça líquida in natura pode ser usada para alimentar coelhos a $87,8 \mathrm{~g}$ por quilograma de dieta. A inclusão de vinhaça autoclavada promove maior taxa e ampliação da digestão in vitro, com reflexos na atividade cecal (Ferreira et al., 2017). Ferreira et al. (2015) concluíram que a inclusão de vinhaça promove a melhoria dos valores de energia digestível e proteína digestível do bagaço de cana-de-açúcar in natura. E que a utilização do bagaço de cana-deaçúcar enriquecido com vinhaça em dietas para coelhos em crescimento se mostrou viável, de modo que a inclusão não alterou o ganho de peso diário dos animais e, portanto, o tempo necessário para atingir o peso de abate. Contudo o bagaço de cana-de-açúcar pode ser incluído em um nível de $100 \mathrm{~g} / \mathrm{kg}$ na dieta de coelhos em crescimento sem efeitos adversos no desempenho de crescimento (Ferreira et al., 2017).

\section{Pólen apícola}

O pólen apícola é um produto natural, coletado pelas abelhas das plantas (Sodré et al., 2007). O pólen de abelha é criado no órgão sexual masculino da flor (anteras) com o objetivo de fertilizar o estigma (Hosseini et al., 2016). É a principal fonte de proteínas para as abelhas (Camazine, 1993; Zeedan Kh et al., 2018). Segundo Oliveira et al. (2013) os grãos de pólen e um material fino em forma de pó, são as sementes masculinas de uma flor que foi recolhida pelas abelhas e à qual elementos especiais (como $\mathrm{Fe}$, $\mathrm{Cu}, \mathrm{Zn}, \mathrm{Cr}$ e Mn) das abelhas foram adicionados. As abelhas coletam o pólen e mistura com suas próprias enzimas digestivas. Como a própolis, outros produtos de abelhas bem conhecidos, a composição química exata depende das plantas das quais as abelhas operárias coletam o pólen e podem variar consideravelmente. Rica fonte de proteínas, aminoácidos essenciais, contendo mais de $51 \%$ de ácidos graxos poli-insaturados e 39\% de ácido linolênico, 20\% de ácido palmítico e 13\% de ácido linoleico; mais de 12 vitaminas, 28 minerais, 11 enzimas ou coenzimas, 11 carboidratos, principalmente glicose, frutose e sacarose, além de flavonoides, carotenoides e fitoesteróis (Attia et al., 2011b; Xu et al., 2009)

De acordo com Perić et al. (2009) e Zeedan Kh et al. (2018) o pólen apícola é um recurso interessante, capaz de melhorar o desempenho produtivo, o peso corporal e a redução do consumo diário de ração, resultando em melhor conversão alimentar. Além disso, os parâmetros imunológicos, perfis bioquímicos sanguíneos e funções renais podem ser melhorados. O pólen apícola apresenta diversas propriedades nutricionais e medicinais como uma substância antienvelhecimento e antioxidante para melhorar as funções 
imunológicas e o crescimento do animal, além de melhora a produção de leite e a taxa de sobrevivência da prole (Dias et al., 2013; Tu et al., 2015). Fazayeli-Rad et al. (2015) e Oliveira et al. (2015) concluíram que o pólen pode ser um complemento nutricional para os animais, pois melhora a eficiência do uso de nutrientes, aumentando sua absorção e acelerando o crescimento dos animais, melhorando seu desempenho produtivo capaz de melhorar o status imunológico do animal, sem aumentar o custo final da dieta. Attia et al. (2011a) concluíram que a melhor concentração de pólen em dietas de coelho e de $200 \mathrm{mg} / \mathrm{kg}$ de peso corporal. De fato, nesse nível, tanto os fanfarrões como os coelhos em crescimento melhoraram o ganho de peso e reduzem o consumo de ração, resultando em uma melhor conversão alimentar. Além disso, mostraram uma melhora na qualidade do sêmen e nos perfis bioquímicos sanguíneos. O efeito positivo do pólen de apícola não está dependendo da estação, mesmo se durante o inverno, devido ao alto nível de ingestão de alimentos, a taxa de crescimento de coelhos é maior. Segundo Attia et al. (2015) na alimentação de coelhos o pólen apícola e o mananoligossacarídeos com ou sem própolis melhoraram o desempenho produtivo e reprodutivo do coelho em comparação com o grupo não suplementado. O que indica que o pólen de abelha e o mananoligossacarídeos possam ser usados como suplemento em coelhos.

\section{Considerações finais}

Embora ainda sejam poucos os alimentos alternativos utilizados na nutrição de coelhos no Brasil, atualmente novos ingredientes vêm sendo utilizados em pesquisas, isso se deve ao fato de que a introdução de alimentos alternativos as dietas convencionais podem auxiliar, de forma mínima, na redução dos potenciais poluídos ambientais e na melhora da lucratividade do produtor.

Contudo, devem ser observados possíveis efeitos negativos da presença de fatores anti-nutricionais que podem comprometem o desempenho dos animais bem como influenciar diretamente na digestibilidade e consequentemente no metabolismo.

Portanto, deve-se sempre realizar a composição bromatológicas dos alimentos alternativos que serão utilizados na alimentação cunícula, para posteriormente realizar a introdução destes coprodutos de forma segura nas rações de coelhos.

\section{Referências bibliográficas}

Alnaimy, A., Gad, A. E., Mustafa, M. M., Atta, M. A. A. \& Basuony, H. A. M. (2017). Using of citrus by-products in farm animals feeding. Open Access Journal of Science, 1(3):58-67.

Arruda, A. M. V., Pereira, E. S., Mizubuti, I. Y. \& Silva, L. D. F. (2003). Importância da fibra na nutrição de coelhos. Semina: Ciências Agrárias, 24181-190.

Attia, Y. A., Al-Hanoun, A. \& Bovera, F. (2011a). Effect of different levels of bee pollen on performance and blood profile of New Zealand White bucks and growth performance of their offspring during summer and winter months. Journal of Animal Physiology and Animal Nutrition, 95(1):17-26.

Attia, Y. A., Al-Hanoun, A., Tag El-Din, A. E., Bovera, F. \& Shewika, Y. E. (2011b). Effect of bee pollen levels on productive, reproductive and blood traits of NZW rabbits. Journal of Animal Physiology and Animal Nutrition, 95(3):294-303.

Attia, Y. A., Bovera, F., El-Tahawy, W. S., El-Hanoun, A. M., Al-Harthi, M. A. \& Habiba, H. I. (2015). Productive and reproductive performance of rabbits does as affected by bee pollen and/or propolis, inulin and/or mannan-oligosaccharides. World Rabbit Science, 23(4):273-282.

Bernardino, V. M. P., Rodrigues, P. B., Naves, L. P., Rosa, P. V., Zangeronimo, M. G., Gomide, E. M. \& Alvarenga, R. R. (2014). Content of plasmatic glycerol and activity of hepatic glycerol kinase in broiler chickens fed diets containing different sources and concentrations of glycerin. Journal of Animal Physiology and Animal Nutrition, 98328-337.

Beserra, V. A., Cesar, A. S. \& Peres, A. A. C. (2016). Adoção da glicerina bruta na dieta animal e seu impacto no produto final. Archivos de Zootecnia, 65259-266.

Camazine, S. (1993). The regulation of pollen foraging by honey bees: how foragers assess the colony's need for pollen. Behavioral Ecology and Sociobiology, 32(4):265-272.

Cheeke, P. R. (1995). Alimentación y nutrición del conejo. Zaragoza: Acribia. 
Coelho, C. C. G. M., Ferreira, W. M., Mota, K. C. N., Rocha, L. F., Sousa, T. N., Costa Júnior, M. B. \& Ferreira, F. N. A. (2016). Utilização digestiva e produtiva de dietas semi-simplificadas com fenos enriquecidos com vinhaça para coelhos em crescimento. Boletim de Indústria Animal, 731-8.

Cruz, O. T. B., Valero, M. V., Zawadzki, F., Rivaroli, D. C., Prado, R. M., Lima, B. S. \& Prado, I. N. (2014). Effect of glycerine and essential oils (Anacardium occidentale and Ricinus communis) on animal performance, feed efficiency and carcass characteristics of crossbred bulls finished in a feedlot system. Italian Journal of Animal Science, 13(4):790-797. doi: http://dx.doi.org/10.4081/ijas.2014.3492.

Dávila, N. F. P., Gomes, A. V. C., Pessôa, M. F., Crespi, M. P. L. \& Crespi Coll, J. F. (2007). Substituição do farelo de soja por farelo de algodão na alimentação de coelhos em crescimento. Acta Scientiarum. Animal Sciences, 29(3):277-282.

De Blas, C. \& Wiseman, J. (2010). Nutrition of the Rabbit. Cambridge: CABI.

De Blas, J. C., Ferrer, P., Rodríguez, C. A., Cerisuelo, A., García-Rebollar, P., Calvet, S. \& Farias, C. (2018). Nutritive value of citrus co-products in rabbit feeding. World Rabbit Science, 26(1):7-14.

Dias, D. M. B., Oliveira, M. C., Silva, D. M., Bonifácio, N. P., Claro, D. d. C. \& Marchesin, W. A. (2013). Bee pollen supplementation in diets for rabbit does and growing rabbits. Acta Scientiarum. Animal Sciences, 35(4):425-430.

Dozier, W. A., Kerr, B. J. \& Corzo, A. (2008). Apparent metabolizable energy of glycerin for broiler chickens. Poultry Science, 87317-322.

Eiras, C. E., Barbosa, L. P., Marques, J. A., Lima, B. S., Zawadzki, F., Perotto, D. \& Prado, I. N. (2014). Glycerine levels in the diets of crossbred bulls finished in feedlot: apparent digestibility, feed intake and animal performance. Animal Feed Science and Technology, 197222-226. doi: http://dx.doi.org/10.1016/j.anifeedsci.2014.07.004.

Eiras, C. E., Marques, J. A., Torrecilhas, J. A., Zawadzki, F., Moletta, J. L. \& Prado, I. N. (2013). Glycerin levels in the diets of crossbred bulls finished in feedlot: ingestion behavior, feeding intake and ruminal efficiency. Acta Scientiarum Animal.Sciences, 35(4):411-416. doi: http://dx.doi.org/10.4025/actascianimsci.v35i4.19090.

Fazayeli-Rad, A. R., Afzali, N., Farhangfar, H. \& Asghari, M. R. (2015). Effect of bee pollen on growth performance, intestinal morphometry and immune status of broiler chicks. European Poultry Science, 791-9.

Ferreira, F. N. A., Ferreira, W. M., Mota, K. C. N., Silva Neta, C. S., Lara, L. B. \& Santos, E. A. (2015). Avaliação nutricional do bagaço de cana-de-açúcar enriquecido com vinhaça em dietas para coelhos em crescimento. Revista Caatinga, 28217-226.

Ferreira, F. N. A., Ferreira, W. M., Silva Neta, C. S., Inácio, D. F. S., Mota, K. C. N., Costa Júnior, M. B. \& Fontes, O. (2017). Effect of dietary inclusion of dried or autoclaved sugarcane bagasse and vinasse on live performance and in vitro evaluations on growing rabbits. Animal Feed science and Technology, 23087-95.

Françozo, M. C., Prado, I. N., Cecato, U., Valero, M. V., Zawadzki, F., Ribeiro, O. L. \& Visentainer, J. V. (2013). Growth performance, carcass characteristics and meat quality of finishing bulls fed crude glycerin-supplemented diets. Brazilian Archives of Biology and Technology, 56(2):327-336.

Galan, G. L., Franco, M. L. R. S., Scapinello, C., Oliveira, E. R. N., Souza, E. D., Gasparino, E. \& Del Vesco, A. P. (2013). Farinha de carcaça de tilápia do Nilo em dietas para coelhos: desempenho e lipídeos séricos. Scientia Agraria Paranaensis, 12193-204.

Gonçalves, L. M. P., Moreira, I., Pozza, P. C., Carvalho, P. L. O., Toledo, J. B. \& Penuela Sierra, L. M. (2014). Semi purified glycerins in growing and finishing pigs feeding $(30-90 \mathrm{~kg})$. . Revista Brasileira de Saúde e Produção Animal, 15221-226.

Hidalgo, K., Rodríguez, B., Valdivié, M. \& Febles, M. (2009). Utilización de la vinaza de destilería como aditivo para pollos en ceba. Revista Cubana de Ciencia Agrícola, 43(3):281-284.

Hosseini, S. M., Azghandi, M. V., Ahani, S. \& Nourmohammadi, R. (2016). Effect of bee pollen and propolis (bee glue) on growth performance and biomarkers of heat stress in broiler chickens reared under high ambient temperature. Journal of Animal and Feed Sciences, 25(1):45-51.

Ibrahim, M. R., El-Banna, H. M., Omara, I. I. \& Suliman, M. A. (2011). Evaluation of nutritive value of some citrus pulp as feedstuffs in rabbit diets. Pakistan Journal of Nutrition, 10(7):667-674. 
Iñigo, M. A., De Blas, J. C., Cachaldora, P. \& García-Rebollar, P. (2011). Effect of starch substitution with crude glycerol on growing rabbit and lactating doe performance. World Rabbit Science, 19(2):67-74.

Jenkins, J. R. (1999). Feeding recommendations for the house rabbit. The Veterinary Clinics of North America. Exotic Animal Practice, 2(1):143-151.

Klinger, A. C. K., Capitânio, J. R., Toledo, G. S. P., Silva, L. P., Pacheco, P. S., Chimainski, M. \& Rodrigues, M. O. (2015). Inclusão de glicerina bruta em dietas para coelhos em crescimento. Archivos de Zootecnia, 64373-376.

Klinger, A. C. K. \& Toledo, G. S. P. (2016). Ingredientes não convencionais na nutrição cunícula no Brasil - Uma revisão. Revista Brasileira de Cunicultura, 91-16.

Klinger, A. C. K., Toledo, G. S. P., Eggers, D. P., Pretto, A., Chimainski, M. \& Da Silva, L. P. (2014). Casca de soja em dietas para coelhos em crescimento. Ciência Rural, 4598-103.

Lehninger, N. D. L. (2006). Principios de bioquímica. São Paulo.

Liao, K., Cai, J., Shi, Z., Tian, G., Yan, D. \& Chen, D. (2017). Effects of raw material extrusion and steam conditioning on feed pellet quality and nutrient digestibility of growing meat rabbits. Animal Nutrition, 3151-155.

Lu, J., Long, X., He, Z., Shen, Y., Yang, Y., Pan, Y., . . Li, H. (2018). Effect of dietary inclusion of dried citrus pulp on growth performance, carcass characteristics, blood metabolites and hepatic antioxidant status of rabbits. Journal of Applied Animal Research, 46(1):529-533.

Luciano, A. P., Lima, G. O. S., Fonseca, L. S. \& Naves, L. P. (2017). Glicerina na alimentação de coelhos. Revista Brasileira de Cunicultura, 129-14.

Machado, L. C. \& Ferreira, W. M. (2012). Atualidades em nutrição de coelhos: 2006 a 2011. Revista Brasileira de Cunicultura, Bambuí, 1(1):17-27.

Machado, L. C. \& Ferreira, W. M. (2014). Opinião: Organização e estratégias da cunicultura brasileirabuscando soluções. Revista Brasileira de Cunicultura, 6(01):1-31.

Machado, L. C. \& Ferreira, W. M. (2016). Utilização de dietas de alto conteúdo de volumosos sobre o peso dos órgãos do sistema digestório em coelhos. Veterinária e Zootecnia, 23(4):664-671.

Maria, B. G., Scapinello, C., Oliveira, A. F. G., Monteiro, A. C., Catelan, F. \& Figueira, J. L. (2013). Digestibilidade da polpa cítrica desidratada e efeito de sua inclusão na dieta sobre o desempenho de coelhos em crescimento. Acta Scientiarum. Animal Sciences, 35(1):85-92.

Martins, P. C., Oliveira, M. C., Silva, D. M., Mesquita, S. A., Oliveira, H. C. \& Marchesin, W. A. (2017). Use of liquid vinasse as a feed additive for Japanese quails. Revista Colombiana de Ciencias Pecuarias, 30(4):278-285.

Molina, E., González Redondo, P., Moreno Rojas, R., Montero Quintero, K., Bracho, B. \& Sánchez Urdaneta, A. (2015). Effects of diets with Amaranthus dubius Mart. ex Thell. on performance and digestibility of growing rabbits. World Rabbit Science, 231-9.

Muller, M. \& Prado, I. N. (2004). Metabolismo da pectina em animais ruminantes. Uma revisão. Revista Varia Scientia, 4(8):45-56.

Nicodemus, N., García, J., Carabaño, R. \& De Blas, J. C. (2007). Effect of substitution of a soybean hull and grape seed meal mixture for traditional fiber sources on digestion and performance of growing rabbits and lactating does. Journal of Animal Science, 85(1):181-187.

Oliveira, M. C., Loch, F. C., Montes, D. S., Martins, P. C., Teixeira, A. S. \& Claro, D. C. (2015). Uso del polen de abeja en la alimentación de pollos de engorda. Revista Mexicana de Ciencias Pecuarias, 6(3):263-276.

Oliveira, M. C. \& Pádua, G. T. (2010). Restrição alimentar em coelhos em crescimento-Revisão de literatura. PUBVET, 4(Art. 730-737):1-13.

Oliveira, M. C., Silva, D. M., Loch, F. C., Martins, P. C., Dias, D. M. B. \& Simon, G. A. (2013). Effect of bee pollen on the immunity and tibia characteristics in broilers. Brazilian Journal of Poultry Science, 15(4):323-327.

Paim, T. P., Louvadini, H., McManus, C. M. \& Abdalla, A. L. (2010). Uso de subprodutos do algodão na nutrição de ruminantes. Ciência Veterinária nos Trópicos, 1324-37. 
Paula, E., Ferreira, W. M., Ferreira, F. N. A., Costa Júnior, M. B., Neves Mota, K. C. n. \& Rocha, L. F. (2016). Digestibilidade e contribuição da cecotrofia de coelhos alimentados com ou sem óleo vegetal na dieta. PUBVET, 11207-312.

Perić, L., Žikić, D. \& Lukić, M. (2009). Application of alternative growth promoters in broiler production. Biotechnology in Animal Husbandry, 25(5-6-1):387-397.

Retore, M., Scapinello, C., Moreira, I., Araujo, I. G., Ponciano Neto, B., Stanquevis, C. \& Oliveira, A. F. G. (2012a). Glicerina semipurificada vegetal e mista na alimentação de coelhos em crescimento. Arquivo Brasileiro de Medicina Veterinária e Zootecnia, 641723-1731.

Retore, M., Scapinello, C., Murakami, A. E., Araujo, I. G., Ponciano Neto, B., Felssner, K. d. S., . . . Oliveira, A. F. G. (2012b). Nutritional evaluation of vegetable and mixed crude glycerin in the diet of growing rabbits. Revista Brasileira de Zootecnia, 41(2):333-340.

Retore, M., Silva, L. P., Toledo, G. S. P., Araújo, I. G. \& Eggers, D. P. (2010). Fontes de fibra de coprodutos agroindustriais proteicos para coelhos em crescimento. Ciência Rural, 40963-969.

Rostagno, H. S., Albino, L. F. T., Donzele, J. L., Gomes, P. C., Oliveira, R., Lopes, D. C. \& Euclides, R. F. (2017). Composição de alimentos e exigências nutricionais (3 ed. Vol. 1). Viçosa: Universidade Federal de Viçosa.

Salvador, A., Igual, M., Contreras, C., Martínez-Navarrete, N. \& Camacho, M. d. M. (2014). Effect of the inclusion of citrus pulp in the diet of goats on cheeses characteristics. Small Ruminant Research, 121(2-3):361-367.

Santos, M. J. B., Ludke, M. C. M. M., Ludke, J. V., Torres, T. R., Lopes, L. d. S. \& Brito, M. S. (2013). Composição química e valores de energia metabolizável de ingredientes alternativos para frangos de corte. Ciência Animal Brasileira, 14(1):32-40.

Shang, S. M., Wu, Z. Y., Liu, G. Y., Sun, C. R., Ma, M. W. \& Li, F. C. (2017). Effect of substituting guinea grass with soybean hulls on production performance and digestion traits in fattening rabbits. World Rabbit Science, 25(3):241-249.

Simon, A., Bergner, H. \& Schwabe, M. (1996). Glycerol as a feed ingredient for broiler chickens. Archives of Animal Nutrition, 49103-112.

Sodré, G. S., Marchini, L. C., De Carvalho, C. A. \& Moreti, A. C. (2007). Pollen analysis in honey samples from the two main producing regions in the Brazilian northeast. Anais da Academia Brasileira de Ciências, 79(3):381-388.

Toledo, G. S. P., Eggers, D. P., da Silva, L. P., Pacheco, P. S., Klinger, A. C. K., Capitânio, J. R. \& Ortiz, J. (2012). Casca de soja em substituição ao feno de alfafa em dietas fareladas para coelhos em crescimento. Ciência Rural, 42(10):1896-1900.

Tu, Y., Zhang, G.-F., Deng, K.-D., Zhang, N.-F. \& Diao, Q.-Y. (2015). Effects of supplementary bee pollen and its polysaccharides on nutrient digestibility and serum biochemical parameters in Holstein calves. Animal Production Science, 55(10):1318-1323.

Valentim, J. K., Machado, L. C., Lopes, V. L., Luana, K., Paula, C., Bittencourt, T. M. \&Dallago, G. M. (2018). Perfil dos criadores de coelho PET no Brasil. Revista Brasileira de Cunicultura, 141-19.

Xu, X., Sun, L., Dong, J. \& Zhang, H. (2009). Breaking the cells of rape bee pollen and consecutive extraction of functional oil with supercritical carbon dioxide. Innovative Food Science \& Emerging Technologies, 10(1):42-46.

Zambom, M. A., Santos, G. T., Modesto, E. C., Alcalde, C. R., Gonçalves, G. D., Silva, D. C., . . Faustino, J. O. (2001). Valor nutricional da casca do grão de soja, farelo de soja, milho moído e farelo de trigo para bovinos. Acta Scientiarum. Animal Sciences, 23(4):937-943.

Zeedan Kh, I. I., Battaa, A. M. E.-N., Abuoghaba, A. A. A. A. \& El-Kholy, K. H. (2018). Effect of bee pollen at different levels as natural additives on immunity and productive performance in rabbit males. Egyptian Poultry Science Journal, 37(1):213-231.

Recebido: 24 de julho, 2019.

Aprovado: 6 de setembro, 2019.

Publicado: 17 de janeiro, 2020.

Licenciamento: Este artigo é publicado na modalidade Acesso Aberto sob a licença Creative Commons Atribuição 4.0 (CC-BY 4.0), a qual permite uso irrestrito, distribuição, reprodução em qualquer meio, desde que o autor e a fonte sejam devidamente creditados. 